University of Wollongong

Research Online

Faculty of Engineering - Papers (Archive)

Faculty of Engineering and Information

Sciences

$1-1-2007$

\title{
Disassembly sequence generation in recycling based on parts accessibility and end-of-life strategy
}

Jing Dong

University of Wollongong, jd58@uow.edu.au

Peter R. Gibson

University of Wollongong, peterg@uow.edu.au

Guenter Arndt

University of Wollongong, garndt@uow.edu.au

Follow this and additional works at: https://ro.uow.edu.au/engpapers

Part of the Engineering Commons

https://ro.uow.edu.au/engpapers/1272

\section{Recommended Citation}

Dong, Jing; Gibson, Peter R.; and Arndt, Guenter: Disassembly sequence generation in recycling based on parts accessibility and end-of-life strategy 2007, 1079-1086.

https://ro.uow.edu.au/engpapers/1272

Research Online is the open access institutional repository for the University of Wollongong. For further information contact the UOW Library: research-pubs@uow.edu.au 


\title{
Disassembly sequence generation in recycling based on parts accessibility and end-of-life strategy
}

\author{
J Dong, P Gibson*, and G Arndt \\ Faculty of Engineering, University of Wollongong, New South Wales, Australia
}

The manuscript was received on 26 July 2006 and was accepted after revision for publication on 21 February 2007.

DOI: 10.1243/09544054JEM697

\begin{abstract}
Nowadays, global sustainability is the central issue in recycling and, increasingly, in manufacturing. Recycling used products can save energy, natural resources, and landfill space, and can reduce air pollution. It can give used products new lives. The study of disassembly is needed in order to make recycling economical, and disassembly sequence generation (DSG) plays an important role. An appropriate disassembly process plan can minimize the cost spent on the disassembly processes and maximize the benefits coming from the reused components. In the current paper, a new approach using Petri net modelling to generate an optimal disassembly sequence (ODS), based on accessibility and end-of-life (EOL) strategy, is described. The different life spans of the reusable components affect the disassembly order, especially in destructive disassembly, and the influence of components with different life spans on DSG is analysed. First, AND/OR graphs are used to generate all feasible disassembly sequences, and then AND/OR graphs are transferred into Petri net graphs while accessibility values and life span values of components are taken into account to obtain the ODS. A program using Microsoft $\mathrm{C}^{++}$is developed to generate the ODS. The disassembly of a C-clamp is used as a trial example.
\end{abstract}

Keywords: sustainability, disassembly, Petri net modelling

\section{INTRODUCTION}

Over the last decade, global sustainability issues have increasingly influenced the reuse of products. Maximizing the amount of recycled components and minimizing the amount of waste pouring into landfills are becoming increasingly important for designers and manufacturers both in manufacturing and demanufacturing. On the other hand, social and environmental awareness has aroused customers' interest in 'green products' and promoted their responsibility to the environment while using products. The more recycled products that are used, the friendlier will be the environment of the future. Disassembly is a key issue in recycling, and makes it possible to extract hazardous substances out of the systems, to reutilize valuable components and materials in products, and to conserve energy, material resources, and landfill capacity.

\footnotetext{
*Corresponding author: Faculty of Engineering, University of Wollongong, Wollongong, New South Wales, Australia. email: peterg@uow.edu.au
}

\subsection{Literature survey}

Research on disassembly process planning has bloomed over the last decade. In the field of disassembly sequence generation (DSG), there are three main categories.

1. Graph-based approaches. Graph theory has been used as a powerful tool to solve the problems in DSG. A number of papers transform the disassembly problems into the graphical representations, e.g. component-fasten graph [1] and AND/OR graph [2]. Li et al. [3] generate a possible multiple components disassembly sequence for maintenance using a disassembly constraint graph. Jiang et al. [4] carry on a research on DSG algorithm based on a hierarchy network graph.

2. Petri net-based approach. Petri nets provide a very promising mathematical method in DSG. Zussman et al. [5] propose a complete and mathematically sound disassembly Petri net (DPN) approach to model and plan disassembly processes. Moore et al. [6] develop an algorithm for automatically generating a DPN from a disassembly precedence 
matrix. Suzuki et al. [7] propose a learning control system for disassembly task sequences modelled by means of Petri nets. This system consists of a supervisory control subsystem and a learning automata system, which allows a flexible design to accommodate frequent changes of manufacturing requirements. Zhao and $\mathrm{Li}[\mathbf{8}]$ use fuzzy reasoning Petri nets to determine disassembly sequence of products.

3. Geometry-based approaches. Srinivasan and Gadh [9] propose a 'wave propagation' approach to reduce the complexity for optimal selective DSG. Shyamsundar et al. [10] use two geometric abstractions to support disassembly sequence analysis: the assembly topology graph and the set of boundary components.

A comprehensive literature review by the authors on DSG and computer-aided design for disassembly was published in 2003 [11]. The present work focuses on hybrid knowledge-based DSG under real failure predicting conditions based on improved accessibility and end-of-life (EOL) strategy.

There are still other approaches to generate disassembly sequences. Gungor and Gupta [12] use a 'dynamic approach' looking at the several factors that can induce uncertainty in disassembly processes. Pan and Duan [13] use a mathematical model of hierarchical fuzzy cognitive map (HFCM) to obtain the disassembly sequences. An approach to facilitate the determination of the disassembly sequence of used products based on component accessibility is proposed by Hsu and Lin [14]. Lee and Moradi [15] propose another approach to determine the disassembly sequence by using a force flow network. A case-based reasoning method is described by Zeid et al. [16] to solve planning for disassembly problems and a computational way for designing assembly with a built-in disassembly pathway that maximizes the profit of disassembly, while satisfying regulatory requirements for component retrieval is represented by Takeuchi and Saitou [17]. Kara et al. [18] develop a selective disassembly methodology for generating an optimum disassembly sequence for EOL products. Torres et al. [19] propose an automatic personal computer (PC) disassembly method for component recovery.

\subsection{Research significance}

Increasing environmental concerns about the disposal of used products have resulted in much effort to consider recycling of products via demanufacturing. Recycling technologies and their design practices will allow manufacturers to minimize waste and to turn waste into profitable products. Disassembly process planning is playing a critically important role in demanufacturing. Its design and improvement is of great significance to achieve the best use of resources (labour, money, and time). DSG is the main task in disassembly process planning. The current paper proposes a new approach to generate optimal disassembly sequences (ODSs) based on accessibility and EOL strategy, and thus contributes towards the economic sustainability of product recycling.

In the present paper, $\mathrm{AND} / \mathrm{OR}$ graphs are used to generate all feasible disassembly sequences. AND/OR graphs are then transferred into Petri net graphs in order to obtain the ODS based on accessibility and EOL strategy. Lifespan theory is first applied to Petri net models to maximize resource recycling in the disassembly process. Petri nets are a tool for system study and modelling. Petri net theory and graphs can be used to model a disassembly operation in order to obtain the best disassembly sequence for a used product.

There are a number of papers that use the Petri net approach. However, using accessibility and EOL strategy to determine the transition values in the Petri net model in order to obtain the optimal disassembly sequence is a new idea. To the current authors' knowledge, there are no papers that discuss this method.

To illustrate the proposed methodology, the dismantling of a C-clamp is described in the present paper, for which a program written in Microsoft Visual $\mathrm{C}++$ to obtain the ODS has been developed.

\subsection{DSG process}

The illustrative C-clamp, shown in Fig. 1, consists of four pieces: $\mathrm{c}_{1}, \mathrm{c}_{2}, \mathrm{c}_{3}$, and $\mathrm{c}_{4}$. First of all, AND/OR net graph of the C-clamp is generated. Then, Petri net graphs are produced by adding some constraints. Finally, an ODS is obtained based on accessibility and EOL strategy.

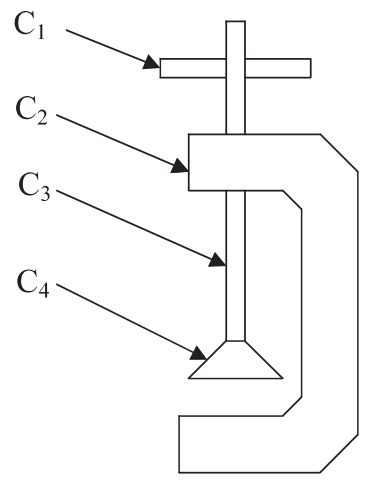

Fig. 1 C-clamp 


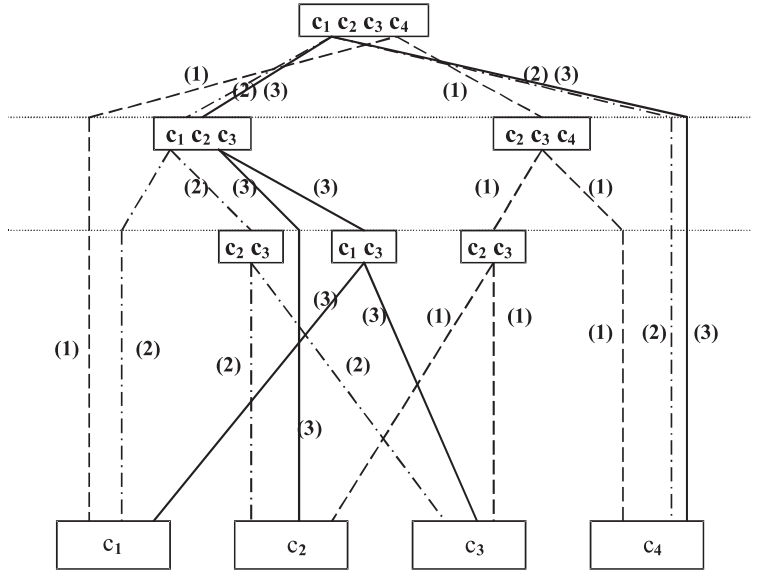

Fig. 2 AND/OR structure net of the C-clamp

\section{GENERATION OF AND/OR NET GRAPHS FOR THE C-CLAMP}

AND/OR net graphs can be used to obtain all the feasible disassembly sequences. Generating an AND/OR net graph only needs to take into consideration the liaison relationship of each part of the C-clamp (see Fig. 2). The graph shows all possibilities for disassembling the C-clamp. Generating all possible disassembly sequences and representing them by these diagrams, makes the process transparent and easy to investigate.

The theoretical number of disassembly sequences can be calculated by using the equation, $S_{n}=n ! / 2$ ( $n$ is the number of components in an assembly). However, the possible sequence number $S_{n}^{\prime}$, which might be executed, is less than $S_{n}$. Some theoretical operations might not be carried out owing to lack of physical relations among the components, or the joins are fixed and no physical separations are possible (e.g. welding). In this case, disassembly is not the reverse of assembly. For example, in the disassembly of the C-clamp, $n=4$. The theoretical sequence number $S_{n}=4 ! / 2=12$; however, the possible sequence number $S_{n}^{\prime}=3$.

The three possible disassembly operation sequences (PDOSs) are shown in Fig. 2, and are labelled (1), (2), and (3).

\section{GENERATION OF PETRI NET GRAPHS FOR THE C-CLAMP DISASSEMBLY}

\subsection{Modelling the disassembly process using Petri net graphs for the C-clamp}

Three PDOSs described by AND/OR net graphs are obtained in the stage described above. Then, AND/OR graphs are converted into Petri net graphs in order to determine the ODS by considering the combination transition value of accessibility and EOL strategy.
Figure 3 is a typical Petri net graph that represents the PDOSs for the C-clamp. A Petri net structure consists of places and transitions. Corresponding to these, a Petri net graph has two types of nodes: A 'circle' $\bigcirc$ represents a place; a 'bar' | represents a transition. In Fig. 3, $t_{i}(i=1,2, \ldots, 8)$ stands for transitions and $p_{i}(i=1,2, \ldots, 10)$ stands for places.

\subsection{Application of Petri net theory to the disassembly process modelling for the C-clamp}

A formal definition of the Petri net structure in the above disassembly is as follows [20]

$C=(\boldsymbol{P}, \boldsymbol{T}, \boldsymbol{I}, \boldsymbol{O})$

$\boldsymbol{P}=\left\{p_{1}, p_{2}, \ldots, p_{n}\right\}$ is a finite set of places;

$\boldsymbol{T}=\left\{t_{1}, t_{2}, \ldots, t_{s}\right\}$ is a finite set of transitions, $\boldsymbol{P} \cup \boldsymbol{T} \neq \phi, \boldsymbol{P} \cap \boldsymbol{T}=\phi, \phi$ is a zero matrix;

$\boldsymbol{I}: \boldsymbol{P} \times \boldsymbol{T} \rightarrow\{0,1\}$ is an input function that defines the set of directed arcs from $\boldsymbol{P}$ to $\boldsymbol{T}$, where $\boldsymbol{I}_{i j}=1$, if $p_{i}$ is in an input place of $t_{j}$, and otherwise, 0 ;

$O: P \times \boldsymbol{T} \rightarrow\{0,1\}$ is an output function that defines the set of directed arcs from $\boldsymbol{T}$ to $\boldsymbol{P}$, where $\boldsymbol{O}_{i j}=1$, if $p_{i}$ is in an output place of $t_{j}$, and otherwise, 0 .

A 'marked' Petri net is defined as

$M=(C, \boldsymbol{\mu})=(\boldsymbol{P}, \boldsymbol{T}, \boldsymbol{I}, \boldsymbol{O}, \boldsymbol{\mu})$

$\boldsymbol{\mu}: \boldsymbol{P} \rightarrow N$ is a marking vector whose $i$ th component represents the number of tokens in the $i$ th place. A token is the condition of an event.

$\boldsymbol{I}=\boldsymbol{P} \times \boldsymbol{T}=\left[\begin{array}{llllllll}1 & 1 & 0 & 0 & 0 & 0 & 0 & 0 \\ 0 & 0 & 1 & 1 & 0 & 0 & 0 & 0 \\ 0 & 0 & 0 & 0 & 1 & 0 & 0 & 0 \\ 0 & 0 & 0 & 0 & 0 & 1 & 0 & 0 \\ 0 & 0 & 0 & 0 & 0 & 0 & 1 & 0 \\ 0 & 0 & 0 & 0 & 0 & 0 & 0 & 1 \\ 0 & 0 & 0 & 0 & 0 & 0 & 0 & 0 \\ 0 & 0 & 0 & 0 & 0 & 0 & 0 & 0 \\ 0 & 0 & 0 & 0 & 0 & 0 & 0 & 0 \\ 0 & 0 & 0 & 0 & 0 & 0 & 0 & 0\end{array}\right]$

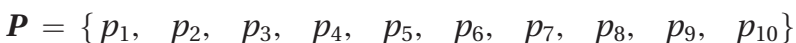




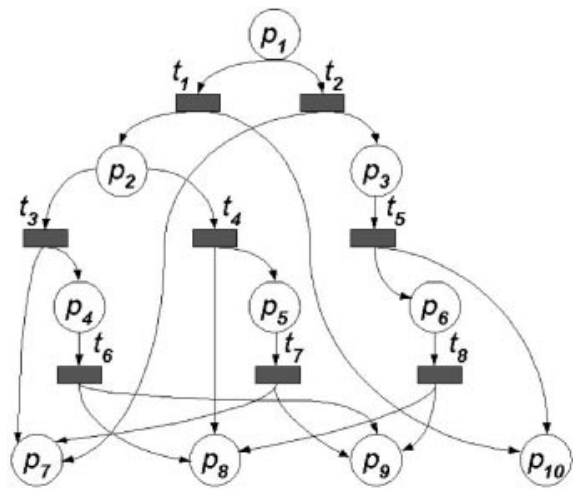

Fig. 3 Petri net graph of the C-clamp to illustrate the places and transitions

$\boldsymbol{T}=\left\{t_{1}, \quad t_{2}, \quad t_{3}, \quad t_{4}, \quad t_{5}, \quad t_{6}, \quad t_{7}, \quad t_{8}\right\}$

$\boldsymbol{I}\left(t_{1}\right)=\left\{p_{1}\right\} \quad \boldsymbol{O}\left(t_{1}\right)=\left\{p_{2}, \quad p_{10}\right\}$

$\boldsymbol{I}\left(t_{2}\right)=\left\{p_{1}\right\} \quad \boldsymbol{O}\left(t_{2}\right)=\left\{p_{3}, \quad p_{7}\right\}$

$\boldsymbol{I}\left(t_{3}\right)=\left\{p_{2}\right\} \quad \boldsymbol{O}\left(t_{3}\right)=\left\{p_{4}, \quad p_{7}\right\}$

$\boldsymbol{I}\left(t_{4}\right)=\left\{p_{2}\right\} \quad \boldsymbol{O}\left(t_{4}\right)=\left\{p_{5}, \quad p_{8}\right\}$

$\boldsymbol{I}\left(t_{5}\right)=\left\{p_{3}\right\} \quad \boldsymbol{O}\left(t_{5}\right)=\left\{p_{6}, \quad p_{10}\right\}$

$\boldsymbol{I}\left(t_{6}\right)=\left\{p_{4}\right\} \quad \boldsymbol{O}\left(t_{6}\right)=\left\{p_{8}, \quad p_{9}\right\}$

$\boldsymbol{I}\left(t_{7}\right)=\left\{p_{5}\right\} \quad \boldsymbol{O}\left(t_{7}\right)=\left\{p_{7}, \quad p_{9}\right\}$

$\boldsymbol{I}\left(t_{8}\right)=\left\{p_{6}\right\} \quad \boldsymbol{O}\left(t_{8}\right)=\left\{p_{8}, \quad p_{9}\right\}$

\section{GENERATION OF THE ODS BASED ON ACCESSIBILITY AND EOL STRATEGY}

When choosing the most appropriate disassembly sequence alternative, many operations can be omitted if they are not profitable, or for other reasons. From the variety of disassembly sequences, the best one should be found to disassemble the C-clamp by determining the most effective and economical sequence, based on accessibility and EOL. By means of Petri net theory, the quantitative analysis for optimal sequence is realized.

In the Petri net structure, a transmission value $M(t)$ represents the 'gain' from the disassembly process. $M(t)$ depends on the values of accessibility and lifespan for components.

For every possible operation sequence, $M_{\mathrm{t}}=$ $\sum M(t), M(t)=m($ accessibility $)+m(\mathrm{EOL})=m_{\mathrm{a}}+m_{\mathrm{e}}$. The best disassembly operation sequence $M_{\mathrm{m}}$ can be decided with the maximum transition value $M_{\mathrm{m}}=$ $\max \left\{M_{(1)}, M_{(2)}, M_{(3)}, \ldots \ldots M_{(n)}\right\}$, where $n$ is the number of possible operations of the disassembly.
Table 1 Values of $t_{\mathrm{a}}$

\begin{tabular}{|c|c|c|c|}
\hline$\underline{t_{\mathrm{a}}}$ & Value & Reason & Comment \\
\hline$t_{1 \mathrm{a}}$ & 0.5 & $\begin{array}{l}\text { It is ok to take off }\{4\} \\
\text { from }\{1234\} \text { with }\{1\}\end{array}$ & Compare with $t_{5 \mathrm{a}}$ \\
\hline$t_{2 \mathrm{a}}$ & 0.5 & $\begin{array}{l}\text { It is easy to take off }\{1\} \\
\text { from }\{1234\} \text { with }\{4\}\end{array}$ & Compare with $t_{3 \mathrm{a}}$ \\
\hline$t_{3 \mathrm{a}}$ & 0.3 & $\begin{array}{l}\text { It is hard to take off }\{1\} \\
\text { from }\{123\} \text { without }\{4\}\end{array}$ & Refer to $t_{2 \mathrm{a}}$ \\
\hline$t_{4 \mathrm{a}}$ & 0.8 & $\begin{array}{l}\text { It is very easy to take off }\{2\} \\
\text { from }\{123\} \text { with }\{1\}\end{array}$ & Compare with $t_{8 \mathrm{a}}$ \\
\hline$t_{5 \mathrm{a}}$ & 0.1 & $\begin{array}{l}\text { It is very hard to take off }\{4\} \\
\quad \text { from }\{1234\} \text { without }\{1\}\end{array}$ & Refer to $t_{1 \mathrm{a}}$ \\
\hline$t_{6 \mathrm{a}}$ & 0.3 & $\begin{array}{l}\text { It is hard to take off }\{3\} \\
\text { from }\{23\} \text { without }\{1\}\end{array}$ & Compare with $t_{4 \mathrm{a}}$ \\
\hline$t_{7 \mathrm{a}}$ & 0.3 & $\begin{array}{l}\text { It is hard to take off }\{3\} \\
\text { from }\{13\} \text { without }\{2\}\end{array}$ & Compare with $t_{3 \mathrm{a}}$ \\
\hline$t_{8 \mathrm{a}}$ & 0.3 & $\begin{array}{l}\text { It is hard to take off }\{3\} \\
\text { from }\{23\} \text { without }\{1\}\end{array}$ & Refer to $t_{4 \mathrm{a}}$ \\
\hline
\end{tabular}

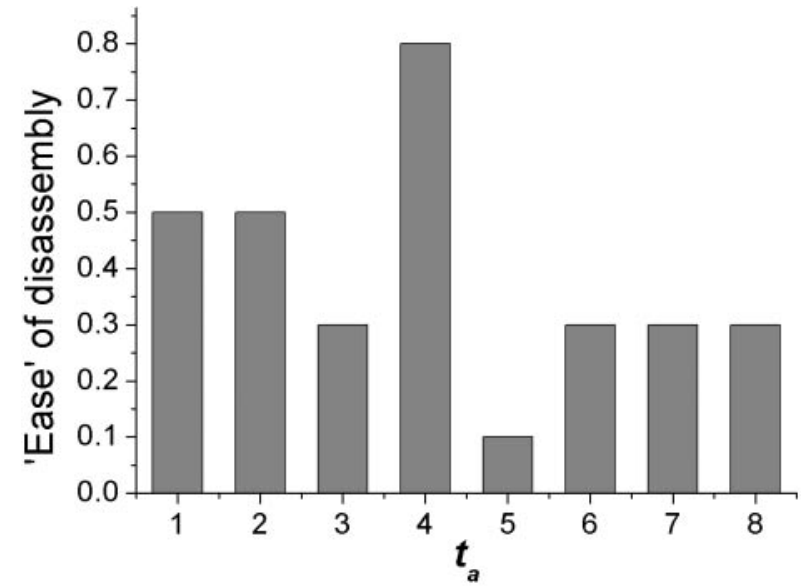

Fig. 4 Values of $t_{\mathrm{a}}$

Regarding the C-clamp, there are three feasible operations - $M_{(1)}, M_{(2)}, M_{(3)}$ - and the best disassembly sequence is $M_{\mathrm{m}}=\max \left\{M_{(1)}, M_{(2)}, M_{(3)}\right\}$.

\subsection{Analysis of the influence of accessibility on the value of $M(t)$}

Accessibility is a 'degree of ease' to take apart an assembly into subassemblies or components. It mainly depends on disassembly forces. There are constraints between components or subassemblies. Higher accessibility means fewer constraints and less force needed to take apart the conjugant. The disassembly order is determined by measuring constraints. The component with minimum constraints is taken away first and that with maximum constraints last.

Assume $m_{\mathrm{a}}=1$ (one) when the easiest disassembly occurs and $m_{\mathrm{a}}=0$ when the most difficult disassembly occurs. Table 1 and Fig. 4 show the allocated values of $t_{\mathrm{a}}$ and the reasons for these. They are 1.0, $0.8,0.5,0.3,0.1$, and 0 . In this step, the values of $t_{\mathrm{a}}$ are decided by analysing the connections one by 
one. The forces that are needed to disassemble connections are mainly considered.

Thus

$$
\begin{aligned}
& \text { Operation (1): } M_{(1) \mathrm{a}}=\sum_{i=2,5,8} t_{(1) \mathrm{ia}}=0.9 \\
& \text { Operation (2): } M_{(2) \mathrm{a}}=\sum_{i=1,3,6} t_{(2) \mathrm{ia}}=1.1 \\
& \text { Operation (3): } M_{(3) \mathrm{a}}=\sum_{i=1,4,7} t_{(3) \mathrm{ia}}=1.6
\end{aligned}
$$

\subsection{Analysis of the influence of EOL strategy on the value of $M(t)$}

A product's lifespan is specified by the lifespan of its main component. The product will be sent to a recycle plant after the main component's lifespan is over. A lot of components can be reused apart from the abandoned components because their lifespans are longer than those of the abandoned components. The different lifespans for the reusable components can affect the disassembly order, especially in destructive disassembly. The components with a short lifespan may be destroyed in order to ensure that the components with a longer lifespan are intact.

Analogous to section 4.1, the conditions of the C-clamp below are used

(a) life spans: $l_{\mathrm{c} 1}=3$ years, $l_{\mathrm{c} 2}=8$ years, $l_{\mathrm{c} 3}=0$ years, $l_{\mathrm{c} 4}=0$ years;

(b) $c_{3}$ and $c_{4}$ are destroyed after being separated because of rust.

Assume $t_{\mathrm{e}}=1$ (one) when there is the longest lifespan; $t_{\mathrm{e}}=0$ when there is the shortest lifespan. Table 2 and Fig. 5 show the allocated values of $t_{\mathrm{e}}$ and the reasons for these. The lifespans are 1.0, 0.8, $0.5,0.3,0.1$, and 0 . Here, the values of $t_{\mathrm{e}}$ are decided through analysing the connection one by one. $t_{\mathrm{e}}$ represents the benefit for every component after being separated from each other.
Thus

Operation (1): $M_{(1) \mathrm{e}}=\sum_{i=2,5,8} t_{(1) i \mathrm{e}}=1.1$

Operation (2): $M_{(2) \mathrm{e}}=\sum_{i=1,3,6} t_{(2) i \mathrm{e}}=1.1$

Operation (3): $M_{(3) \mathrm{e}}=\sum_{i=1,4,7} t_{(3) i \mathrm{e}}=1.2$

\subsection{Combine influence of accessibility and EOL strategy on the value of $M(t)$}

The ODS is determined by both accessibility and EOL strategy values.

Disassembly gain value:

$$
\begin{aligned}
& M(t)=m(\text { accessibility })+m(\mathrm{EOL})=m_{\mathrm{a}}+m_{\mathrm{e}} \\
& M_{(1)}=M_{(1) \mathrm{a}}+M_{(1) \mathrm{e}}=2.0, \quad M_{(2)}=M_{(2) \mathrm{a}}+M_{(2) \mathrm{e}}=2.2, \\
& M_{(3)}=M_{(3) \mathrm{a}}+M_{(3) \mathrm{e}}=2.8 \\
& \mathrm{M}=\max \left\{M_{(1)}, \quad M_{(2)}, \quad M_{(3)}\right\}=M_{(3)}=2.8
\end{aligned}
$$

\begin{tabular}{|c|c|c|c|}
\hline$\underline{t_{\mathrm{e}}}$ & Value & Reason & Comment \\
\hline$t_{1 \mathrm{e}}$ & 0.1 & $\begin{array}{l}\text { Because of } l_{\mathrm{c} 4}=0 \text {, taking }\{4\} \text { from }\{1234\} \text { cannot bring any benefit, but it is the transition step and the } \\
\text { following steps can bring benefits }\end{array}$ & Lowest value \\
\hline$t_{2 \mathrm{e}}$ & 0.5 & $\begin{array}{l}\text { From this operation, }\{1\} \text { and }\{234\} \text { are obtained. }\{1\} \text { has a short lifespan, but benefits can also be } \\
\text { obtained from the next steps, so the value is medium }\end{array}$ & Medium value \\
\hline$t_{3 \mathrm{e}}$ & 0.5 & $\begin{array}{l}\text { This step is to take }\{1\} \text { from }\{123\} .\{1\} \text { has a short lifespan but benefits can also be obtained from } \\
\text { following steps, so the value is medium }\end{array}$ & Medium value \\
\hline$t_{4 \mathrm{e}}$ & 0.8 & $\begin{array}{l}\text { This step is to take }\{2\} \text { from }\{123\} .\{2\} \text { has the longest lifespan and the biggest benefit, so } t_{4 \mathrm{e}} \text { has } \\
\text { the maximum value }\end{array}$ & Highest value \\
\hline$t_{5 \mathrm{e}}$ & 0.1 & $\begin{array}{l}\text { Because of } l_{\mathrm{c} 4}=0 \text {, taking }\{4\} \text { from }\{234\} \text { cannot bring any benefit, but it is the transition step and } \\
\text { the following steps can bring benefits }\end{array}$ & Lowest value \\
\hline$t_{6 \mathrm{e}}$ & 0.5 & This step is to take $\{3\}$ from $\{23\} .\{3\}$ is unusable and $\{2\}$ has the longest lifespan, so the value is medium & Medium value \\
\hline$t_{7 \mathrm{e}}$ & 0.3 & This step is to take $\{1\}$ from $\{13\} .\{1\}$ has a short lifespan and $\{3\}$ is unusable, so the value is lower & Lower value \\
\hline$t_{8 \mathrm{e}}$ & 0.5 & This step is to take $\{3\}$ from $\{23\} .\{2\}$ has the longer lifespan, but $\{3\}$ is unusable, so the value is medium & Medium value \\
\hline
\end{tabular}

An ODS should possess a maximum benefit from the disassembly operation. Therefore, operation (3) (see Fig. 2) is the best one in this case. A program in

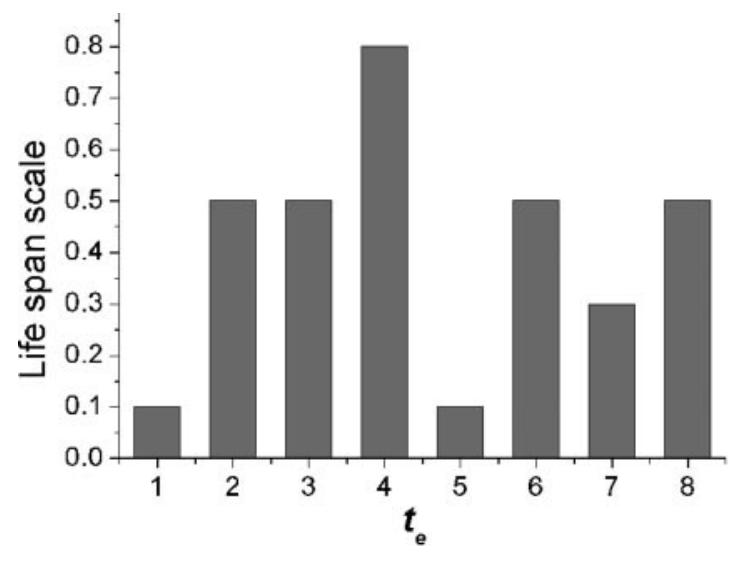

Fig. 5 The value of $t_{\mathrm{e}}$

Table 2 Values of $t_{\mathrm{e}}$ 
Microsoft $\mathrm{C}++$ on calculating $M_{(\mathrm{t})}$ has been developed.

\section{CONCLUSIONS}

Determining the best disassembly sequence is the main aim of disassembly process planning. The best disassembly sequence decides the best order for separating a product into its constituent parts, components, or other groupings. Many new methods have been developed over recent years to generate disassembly sequences and determine the optimal one: this is becoming an increasingly important issue in the study of disassembly.

In the current paper, a new approach has been developed for DSG of used products based on both accessibility and EOL strategy. Petri net models have been presented to obtain the ODS. The DSG of the C-clamp has been illustrated in this paper as an example. The following conclusions are drawn according to the proposed approach.

1. Using AND/OR and Petri net theory to model a disassembly sequence has high potential for research because generating a disassembly process should consider the product topology, mating relations, and precedence relations. AND/OR and Petri net graphs are very suitable to describe these relations.

2. Analysing accessibility means examining every component's constraints coming from the liaison of other joined components. The component with the minimum constraint can first be taken apart and, finally, the component with the maximum constraint.

3. Different components may have different lifespans, which will affect the disassembly orders dramatically.

The proposed approach also provides a base for further study on utilizing the accessibility and EOL strategy in DSG. Further work will focus on: (a) modelling the disassembly process to products with more complicated structures, e.g. a two-shaft helical gearbox consisting of 41 parts, by using extended Petri net theory; (b) determining accessibility values more rationally/accurately by analysing 'decomposition forces', i.e. the external forces applied to components' connections. It is believed that the proposed approach points the way to use lifespan theory to decide the best disassembly sequence for used products.

\section{REFERENCES}

1 Zhang, H. C. and Kuo, T. C. A graph-based approach to disassembly model for end-of-life product recycling. In Proceedings of IEEE/CPMT Symposium on International electronics manufacturing technology, Austin, Texas, 1996, pp. 247-254 (Institute of Electrical and Electronics Engineers, Piscataway, New Jersey, USA).

2 Murayama, T., Kagawa, K., and Oba, F. Computer-aided redesign for improving recyclability. In Proceedings of EcoDesign'99: First International Symposium on Environmentally conscious design and inverse manufacturing, Tokyo, Japan, 1999, pp. 746-751 (IEEE Computer Society Press, California, USA).

3 Li, J. R., Khoo, L. P., and Tor, S. B. Generation of possible multiple components disassembly sequence for maintenance using a disassembly constraint graph. Int. J. Prod. Econ., 2006, 102(1), 51-65.

4 Jiang, J. B., Guo, W. X., Liu, Z. F., and Liu, G. F. Research on disassembly sequence generation algorithm based on hierarchy network graph. Computer Integrated Mfg Systems, 2004, 10(9), 1129-1133.

5 Zussman, E., Zhou, M. C., and Caudill, R. Disassembly Petri net approach to modelling and planning disassembly processes of electronic products. IEEE Proc. Electr. Environ., 1998, 331-336.

6 Moore, K. E., Gungor, A., and Gupta, S. M. Petri net approach to disassembly process planning. Computer Ind. Engng, 1998, 35(1-2), 165-168.

7 Suzuki, T., Zanma, T., Inaba, A., and Okuma, S. Learning control of disassembly Petri net - an approach with discrete event system theory. In Proceedings of IEEE International Conference on Robotics and automation, Minneapolis, USA, pp. 84-191 (IEEE Computer Society Press, California, USA).

8 Zhao, S. E. and Li, Y. L. Fuzzy reasoning Petri nets and its application to disassembly sequence decision of products. Control Decision, 2005, 20(10), $181-1184$.

9 Srinivasan, H. and Gadh, R. Complexity reduction in geometric selective disassembly using the wave propagation abstraction. In Proceedings of IEEE International Conference on Robotics and automation, Leuven, Belgium, 1998, pp. 1478-1483.

10 Shyamsundar, N. and Gadh, R. Geometric abstractions to support disassembly analysis. IIE Trans., 1999, 31(10), 935-946.

11 Dong, J. and Arndt, G. A review of current research on disassembly sequence generation and computer aided design for disassembly. Proc. Instn Mech. Engrs, Part B: J. Engineering Manufacture, 2003, 217, 299-312.

12 Gungor, A. and Gupta, S. M. Disassembly sequence planning for products with defective parts in product recovery. Computers and Ind. Engng, 1998, 35(1-2), 161-164.

13 Pan, X. and Duan, G. Intelligent disassembly sequence planning for EOL recycling hierarchical fuzzy cognitive map. In Proceedings of IEEE Symposiums of Electronics and the Environment, New Orleans, LA, 2005, pp. 255-259.

14 Hsu, H. Y. and Lin, G. C. I. An accessibility based approach for design for disassembly applications. In Proceedings of 8th ICME International Conference on Manufacturing engineering, Sydney, Australia, 2000, pp. $463-467$. 
15 Lee, S. and Moradi, H. Disassembly sequencing and assembly sequence verification using force flow networks. In Proceedings of IEEE International Conference on Robotics and automation, Detroit, Michigan, USA, 1999, pp. 2762-2767.

16 Zeid, I., Gupta, S. M., and Bardasz, T. A case-based reasoning approach to planning for disassembly. J. Intell. Mfg, 1997, 8, 97-106.

17 Takeuchi, S. and Saitou, K. Design for productembedded disassembly. In Proceedings of IDETC/ CIE ASME 2005 International Design Engineering Technical Conferences and Computers and Information in Engineering Conference (DETC2005-85260), California, USA.

18 Kara, S., Pornprasipol, P., and Kaebernick, H. A selective disassembly methodology for end-of-life products. Assembly Automat., 2005, 25(2), 124-134.

19 Torres, F. et al. Automatic PC disassembly for component recovery. Int. J. Advd Mfg Technol., 2004, 23(1-2), 39-46.

20 Peterson, J. L. Petri net theory and the modelling of systems, 1981 (Prentice-Hall, USA).

\section{APPENDIX}

\section{Notation}

$\mathrm{c}_{i} \quad$ product components

DSG disassembly sequence generation

EOL end-of-life

$\boldsymbol{I}, \boldsymbol{O} \quad$ input and output functions

$M(t) \quad$ transition value

$M_{\mathrm{m}} \quad$ maximum transition value

ODS(s) optimal disassembly sequence

$\boldsymbol{P} \quad$ set of places

PDOS(s) possible disassembly operation sequence(s)

$S_{n} \quad$ theoretical disassembly sequence number

$S_{n}^{\prime} \quad$ possible disassembly sequence number

$t_{\mathrm{a}} \quad$ value of accessibility

$t_{\mathrm{e}} \quad$ value of EOL

$t_{i}, p_{i} \quad$ transitions and places

$\boldsymbol{T} \quad$ set of transitions

$\mu \quad$ marking vector 
Reproduced with permission of the copyright owner. Further reproduction prohibited without permission. 